\title{
A New Look of Dehumanization in Work Domain: The Relationship between Communication Means and Disrespect to Deliveryman
}

\author{
Yingzhi Zhang \\ Shanghai Foreign Language School, Shanghai, China \\ Email: melissazhangyz@outlook.com
}

How to cite this paper: Zhang, Y. Z (2020). A New Look of Dehumanization in Work Domain: The Relationship between Communication Means and Disrespect to Deliveryman. Psychology, 11, 572-580. https://doi.org/10.4236/psych.2020.114038

Received: February 21, 2020

Accepted: March 28, 2020

Published: April 2, 2020

Copyright $\odot 2020$ by author(s) and Scientific Research Publishing Inc. This work is licensed under the Creative Commons Attribution International License (CC BY 4.0).

http://creativecommons.org/licenses/by/4.0/

\section{(c) (i) Open Acces}

\begin{abstract}
The present study provides a new aspect to understand the disrespect to deliveryman. A survey was conducted based on the hypothesis which assumed that the disrespect is caused by the indirect communication via technology, mediated by mechanistic dehumanization. Using cashier as the representative of jobs which adopt traditional face-to-face communication, we tested the dehumanization of deliveryman through four measurements and then tested the disrespect degree by giving an unpleasant scenario. The result suggests that people significantly dehumanize deliveryman more in two measurements. Additionally, the dehumanization degrees of these two measurements are positively correlated with disrespect degrees. In general, the finding provides a new aspect to view the dehumanization in the work domain.
\end{abstract}

\section{Keywords}

Prejudice, Dehumanization, Communication, Computerization, Deliverymen

\section{Introduction}

In the context of China, express logistics experienced rapid development in the past few years. People can get almost everything delivered to one's home now. Deliveryman can be easily found all over the city, from developed metropolises to small towns. Riding an electronic motorcycle, a deliveryman can bring anything from food and clothes to medicine and sex products. Jack Ma, founder of the Alibaba Group once predicted that it would be hard to find couples walking hand in hand in a shopping mall in the future. The convenience and inexpen- 
siveness of express logistics have attracted a large number of customers and E-commerce sellers, and thus have become more and more welcomed by the market.

On the other hand, it has been more frequently reported that deliverymen were treated badly. On Jul. 28, 2017, a deliveryman from ZTO express company was punched to incontinence by a customer just because he was late for about five minutes, reported by news.youth.cn. The videos of similar situations were also widely spread on the Internet, drawing more attention from the public. The previous study (Zhao, 2014) showed that most participants complained about the unpleasant situation by utilizing negative word-of-mouth while the second priority is to complain to the deliveryman directly.

In this paper, we suggest that customers poorly treat the deliveryman mainly because they dehumanize deliveryman. Quoted from the great psychologist Philip G. Zimbardo, "One of the worst things that we can do to our fellow human beings is depriving them of their humanity, rendering them worthless by exercising the psychological process of dehumanization." (Smith \& Zimbardo, 2007). Dehumanization has been everywhere in our life, from abstract artworks to realistic work fields.

The existence of dehumanization could date back to 7 B.C. when ancient Greek poet Simonides described women as animals-like subhuman souls. Later on, this philosophy was developed by Aristotle in about 4 B.C. He claimed that all foreigners were slaves by humans and lacked rationality, which, Aristotle believed, was the key to become a human being (Smith, 2011).

Although many philosophers have long been discussing dehumanization and similar phenomenon, it is widely agreed that modern research on dehumanization started in the late $20^{\text {th }}$ century. Modern social psychologists concluded four major theories on the topic of dehumanization: Infrahumanization Theory, the Dual Model of Dehumanization, the Stereotype Content Account, and the Mind Perception Account (Haslam \& Loughnan, 2014). In particular, the dual model theory (Haslam, 2006) provided a new conceptual model for dehumanization which defined two dimensions of dehumanization: animalistic dehumanization and mechanistic dehumanization. Animalistic dehumanization happens when a person is linked to an animal and denied human uniqueness which includes refinement, self-control, intelligence, and rationality (Leyens, Rodriguez, Rodriguez, Gaunt, Paladino, Vaes et al., 2001). On the other hand, mechanistic dehumanization happens when a person is linked to an inanimate object and denied human nature which includes warmth, emotion, and individuality. In this model, human uniqueness and human nature are two different senses of humanness. Traits that represent human uniqueness are considered to be later developed and could be different according to different cultural environments; traits that represent human nature are considered to be fundamental, universal and related to emotion. This dual model of dehumanization has been widely used in research, and it has been examined to have extensive evidence in both perceivers' 
and targets' perception for groups and individuals. The definition and characteristics of mechanistic dehumanization in the present study are also adopted from this model. The present study adopted the dual model theory as its basis because it clearly elaborates the relationship between human and mechanistic traits which is an essential term in our study.

Dehumanization over certain job types has not been frequently discussed until recent years. Also adopting the dual model theory used in the present study, Andrighetto, Baldissarri, and Volpato (2016) found that laypeople automatically objectified workers, especially those who do repetitive and fragmented work on the assembly lines in factories. The workers were thought by the participants as instrument-like and thus were less able to have human emotions. More recent, Valtorta, Baldissarri, Andrighetto, and Volpato (2019) examined the dehumanization level to certain occupational groups with taint working environments. The result suggested that workers in the physical taint cluster were associated with biological metaphors, workers in the social taint cluster were associated with objects, and workers in the moral taint cluster were associated with animals.

Referring to deliveryman, we supposed that people dehumanize this occupational group because the communication between deliverymen and their customers is exclusively mediated by technology, such as internet, application, phone and etc. Take food delivery as an example. When we order the food online, we either use an app or telephoning the restaurant. Then, the communication during the buying process is completed via electronic devices instead of a face-to-face talk. What the customer needs to do is just wait for the deliveryman and get his or her food. Thus, some people might consider deliveryman as a transportation which the product takes to get to their home.

Although none of the previous study focused directly on the correlation between computerization and its effects on deliverymen, there was a study which focused on the interaction between teachers and students. Nissenbaum and Walker (1998) examined whether computerization of schools dehumanizes education. They did a grounded analysis and concluded that there were valid grounds for concerns about dehumanization arising from the use of computers, but not the most serious risks. Another study (Waytz \& Gray, 2018) examined how technology related to sociability. The result suggested that both positive and negative relationship can be found and both were highly dependent on age, generation and social economic status (SES).

Thus, we assumed that the way people interact with deliveryman, which is exclusively through technology, influences the way people treat them, and mechanistic dehumanization is the mediator. We designed a survey based on this hypothesis, which aims at discovering the cause of disrespect to deliverymen and then providing some suggestions to improve the situation.

The present study used deliveryman as the representative of internet communication jobs; cashier in milk-tea shop as the representative of traditional face-to-face communication jobs. The survey asked the participants to value 
both jobs in four measurements of dehumanization. Furthermore, to test the behaviors of participants, they were randomly assigned to one of the two scenarios which required them to report their reactions when unpleasant accidents happened when buying a beverage.

\section{Method}

\subsection{Participant}

We recruited 218 Chinese participants from Shanghai Jingan Joy City shopping mall $(\mathrm{N}=54)$ and via Wenjuanxing.cn $(\mathrm{N}=164)$, a Chinese online survey website. All participants joined as volunteers. We excluded 3 participants who were not born or did not grow up in China. We also excluded 6 participants who reported that they did not finish the survey seriously.

The final sample of 209 varied in age $(M=33.13, S D=12.36)$ and gender $(67.46 \%$ female). While all of the participants are Chinese, $33.97 \%$ of them $(N=$ 71) reported that they lived in international metropolis; $50.72 \%$ of them $(N=$ 106) lived in other first-tier cities; $6.70 \%$ of them $(N=14)$ lived in second-tier cities; $8.61 \%$ of them $(N=18)$ lived in other smaller cities. We also collected participants' education backgrounds. $27.07 \%$ of the participants $(N=62)$ have a highest degree in high school; $3.49 \%$ of the participants $(N=8)$ have a highest degree in vocational college; $40.61 \%$ of the participants $(N=93)$ have a highest degree in bachelor; $17.03 \%$ of the participants $(N=25)$ have a highest degree in master and $0.87 \%$ of the participants $(N=2)$ have a degree in doctor or higher.

\subsection{Procedure}

The present study was based on a survey that has seven pages in total. We used WJX.CN, which is a professional online survey platform, to design and distribute our survey. Since all the seven pages are highly connected and related to each and another, the participants were required to finish the whole survey without being disrupted. All the participants finished the same survey and we did not conduct any pilot test. Referring to the answers, Likert's scales were used for most key questions.

Participants were informed that they would complete a seven-page survey about job prejudice on the computer. After reading and agreeing to the consent form on the first page, participants were asked if they were born and grown up in China. If they chose "no", they would directly jump to the end of the survey. This question was designed to control the background information and everyday life experience of our participants. The survey was designed under the Chinese cultural context, so we excluded those who were not familiar with the situation in China.

On the second page, participants were asked to fill in some demographic information including age, gender, education background and present address. At the end of the second page, participants were asked to rate the status of several occupations including policeman, teacher, cashier, doctor, deliveryman, and 
shopping assistant (range: 1 - 5, higher score means higher status). This question aimed at testing whether the participants have explicit bias to deliveryman or cashier compared to other jobs.

Then, on page three, participants viewed a picture of a Chinese male deliveryman and were given related information about him ("He is Li Dezheng, a 29-year-old deliveryman."). We instructed them to answer the following six questions based on the picture. In case of intervention, we intentionally hide the facial expressions of the person in the picture so that participants cannot observe his emotion. Four of the questions were designed to measure if the participants had explicit dehumanization over the man in the picture. We asked them to evaluate the importance of punctuality (range: $1-5$, higher score means punctuality is more important), the degree of emotional sensitivity (range: 1 - 5, higher score means more sensitive to emotion), the degree of tiredness (range: 1 - 5, higher score means more tired) and the degree of pain tolerance (range: 1 5 , higher score means bearing more pain) after a day's work. These four measurements were chosen based on the definition and characteristics of mechanistic dehumanization (Haslam, 2006).

On page four, participants viewed another picture of a Chinese male cashier in milk tea shop and were given related information about him ("He is Chen Zhenchao, a 28-year-old cashier in milk-tea shop.") The information was designed to be slightly different so as to be convincing. Then, participants were asked to answer the exactly same six questions as they had done on the previous page. The only difference is that they were rating Mr. Chen on this page. We recoded emotional degree and sensitivity to tiredness for both pages so that these two variables could fit in the scale which higher score means more dehumanization.

Next, each participant was randomly assigned to one of the next two pages by the random screening tool in WJX, which means they would see either page five or page six. The two pages describe two scenarios which were similar. We required them to imagine that they plan to buy themselves a drink during the lunch break after a tiring morning. However, their drink was not only delayed but was also poured over. Then, they were asked to report their reactions to the unpleasant situation in detail. The only difference between the two scenarios is that they buy the drink on a food delivery app in the first scenario but they go out to buy a drink in a real shop in the second one. To put it in another way, a deliveryman offers the drink in page 5 scenario while a cashier offers the drink in page 6 scenario. We recorded all the answers, randomized them and then showed them to three 16-year-old female coders. The coders were required to evaluate the degree of disrespect (range: 1 - 4, higher score means more disrespectful). This test aimed at observing the behavior of people.

In the final page, participants were asked if they answered seriously or not. Then, they were told that all the survey questions are finished and thanked for participation. 
To examine whether the different ways of interacting with the customers, which is presented as different job types in this survey, influenced how much participants mechanistically dehumanized them, we constructed an analysis of variance (ANOVA) with four measurements for explicit dehumanization.

\section{Result and Discussion}

As expected, participants' ratings of "importance of punctuality" were significantly higher for deliveryman $(M=4.58, S D=0.690)$ than cashier $(M=3.76, S D$ $=0.899), F(1,208)=162.493, p<0.001$, suggesting that participants thought being punctual was more important for deliveryman than cashier in a milk-tea shop. Similar to punctuality, participants' ratings of pain tolerance was significantly higher for deliveryman $(M=3.89, S D=0.782)$ than cashier $(M=3.32, S D$ $=0.794), F(1,208)=98.386, p<0.001$, suggesting that participants believed deliveryman were less sensitive to physical hurt or psychological pressure comparing to cashier in milk-tea shop. See Figure 1. In other words, participants mechanistically dehumanized deliveryman significantly more than cashier in the milk-tea shop when using punctuality and pain tolerance as measurements.

As can be seen in Figure 1, however, participants' ratings had no significance for deliveryman and cashier in emotional degree, $F(1,208)=0.224, p>0.05$, suggesting that participants couldn't tell any difference in emotional degree between deliveryman and cashier in milk-tea shop.

Contrary to expectations, in the reversed scale of tiredness measurement, participants' ratings were significantly lower for deliveryman $(M=1.5646, S D=$ $0.71850)$ than cashier $(M=2.3876, S D=0.78326), F(1,208)=206.687, p<$ 0.001 , suggesting that participants assumed cashier felt less tired after whole day's work. In other words, participants mechanistically dehumanized cashier in milk-tea shop significantly more than deliveryman when using a tiredness measurement.

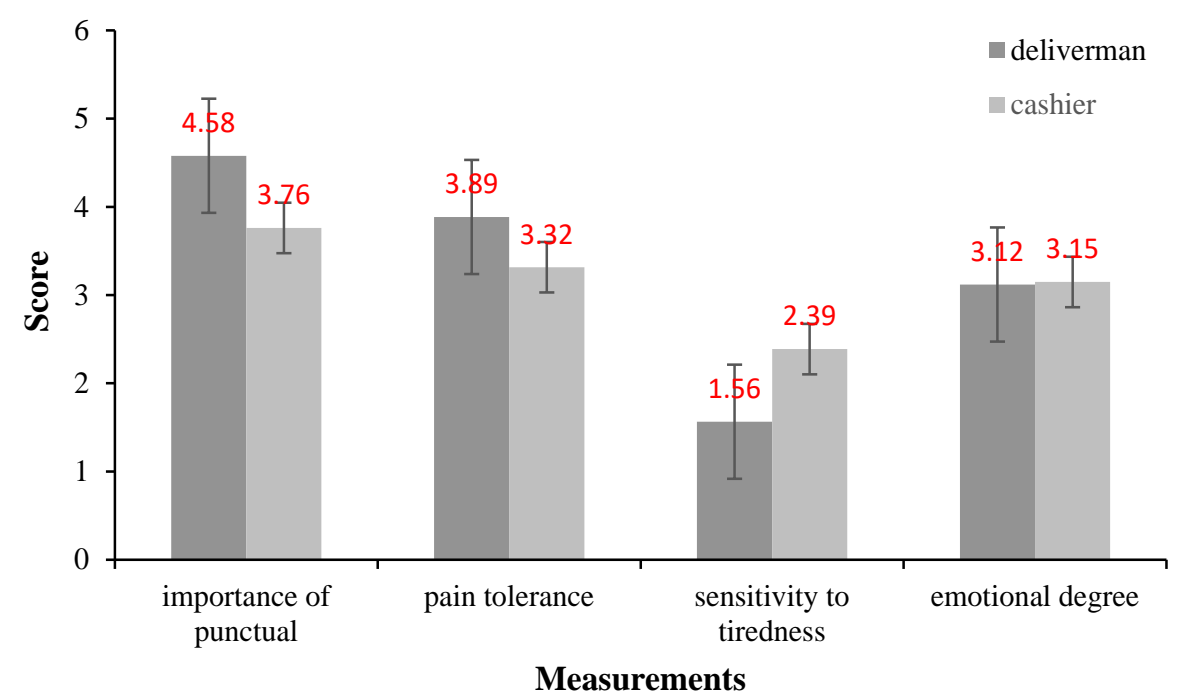

Figure 1. Ratings (estimated means and standard errors) for explicit dehumanization. 
Next, we tested the correlation between dehumanization and participants' disrespect behaviors. We used cashier as the baseline when calculating dehumanization degree. Consistent to hypothesis, explicit dehumanization to deliveryman was positively correlated with disrespect level when using pain tolerance $(r$ $=0.231, p<0.035)$ and importance of punctual $(r=0.205, p<0.017)$ as measurements. To put it in another way, if participants dehumanized deliveryman significantly more, they behave more disrespectfully.

We controlled explicit bias at first. The result of social status comparison showed that there was no significance in explicit bias towards deliveryman and cashier, $F(1,208)=159.133, p=0.089$. Thus, the negative attitude and disrespectful behaviors of participants were not caused by explicit bias due to the social status or occupations.

To put it in another way, participants believed that punctuality and pain tolerance are two important traits of deliverymen. These traits were considered by participants to symbolize both the job itself and the individuals who take this job. Implicitly, participants thought that deliverymen should be more punctual and suffer more painful senses rather than people in other occupations. This also infers that participants would have stricter requirements on deliverymen when they are customers. Thus, the implicit bias would easily cause a less control on the disrespectful behavior, including oral violence and even physical conflicts.

\section{Conclusion and Future Work}

The present study demonstrated that, consistent with the hypothesis, the communication means influenced peoples' behavior to deliveryman. To be more specific, the disrespect is the result of mechanistic dehumanization. However, the sensitivity to tiredness showed an opposite result to the hypothesis and the other two measurements. One possible explanation is that tiredness is more exposed to customers than other measurements. When they shop online, customers are situated in a totally different environment than the deliveryman.

In general, this finding has a wide implication. First, it reveals the fact that people are likely to mechanistically dehumanize deliveryman. The results appeal for more attention to the human nature of deliveryman. Laypeople should consider deliveryman as an occupation instead of a convenient tool. Second, the result provides possible solutions to the logistics industry. The root cause of mechanistic dehumanization is the indirect communication via phone or internet. Thus, changing the way of communication may decrease dehumanization as well as the disrespect. Express companies can add more face-to-face communication opportunities for customers. For example, they can require all the deliveryman to upload their photo. Instead of showing a motorcycle of deliveryman on the app, they can use the photo of deliveryman. Another way to solve the problem is to avoid all the contact between deliveryman and customers by using delivery box. The customers can get the package from the box without telephoning the deliveryman. Third, the relationship between technology and dehumanization 
deserves more study in the future. We are getting in a more computerized world. While technology is connecting people from all over the world, people are also dragged away from real human. The previous study (Waytz \& Gray, 2018) suggested that we are less sensitive to human being while chatting online. Thus, more computerization may cause more mechanistic dehumanization.

The present study has three main shortcomings. First, participants may not give a real answer on survey. We did not adopt an experiment. Instead of finding a real deliveryman and a cashier to act, we just described the scenario with words on survey and instructed the customers to imagine their behavior. This may lead to a dishonest response caused by some moral concerns of the participants. Besides, we tested dehumanization before giving the scenarios, so participants may guess out the purpose of the study and not report the real behavior. Second, the survey did not ask the participants to report their occupation. Instead, we just simply examine whether there is a possible correlation between education background and dehumanization and found no significance. However, the social economic status (SES) and working experience of the participants may also be included as factors. Third, the survey has a total of only 209 samples, which is relatively small.

Future study can recruit more participants and improve the shortcomings of the method of the present study. For example, an Implicit Association Test (IAT) could be used to test the implicit bias more accurately. In addition, future study can also extend the job type to other fields which adopt technology as communication means.

\section{Acknowledgments}

Thanks to experimenters Rui Gu, Tianyi Li, and Youyi Shuai for their considerable efforts, and to Zhenchao Hu for his contributions.

\section{Conflicts of Interest}

The author declares no conflicts of interest regarding the publication of this paper.

\section{References}

Andrighetto, L., Baldissarri, C., \& Volpato, C. (2016). (Still) Modern Times: Objectification at Work: Objectification of Factory Workers. European Journal of Social Psychology, 47, 25-35. https://doi.org/10.1002/ejsp.2190

Haslam, N. (2006). Dehumanization: An Integrative Review. Personality and Social Psychology Review, 10, 252-264. https://doi.org/10.1207/s15327957pspr1003_4

Haslam, N., \& Loughnan, S. (2014) Dehumanization and Infrahumanization. Annual Review of Psychology, 65, 399-423.

https://doi.org/10.1146/annurev-psych-010213-115045

Leyens, J. Ph., Rodriguez, A. P., Rodriguez, R. T., Gaunt, R., Paladino, P. M., Vaes, J. et al. (2001). Psychological Essentialism and the Attribution of Uniquely Human Emotions to In-Groups and Out-Groups. European Journal of Social Psychology, 31, 395-411. 
https://doi.org/10.1002/ejsp.50

Nissenbaum, H., \& Walker, D. (1998). Will Computers Dehumanize Education? A Grounded Approach to Values at Risk. Technology in Society, 20, 237-273. https://doi.org/10.1016/S0160-791X(98)00011-6

Smith, D. L. (2011). Less Than Human: Why We Demean, Enslave, and Exterminate Others. New York: St. Martin's Press.

Smith, M. B., \& Zimbardo, P. (2007). The Lucifer Effect: Understanding How Good People Turn Evil. New York: Random House.

Valtorta, R. R., Baldissarri, C., Andrighetto, L., \& Volpato, C. (2019). Dirty Jobs and Dehumanization of Workers. British Journal of Social Psychology, 58, 955-970. https://doi.org/10.1111/bjso.12315

Waytz, A., \& Gray, K. (2018). Does Online Technology Make Us More or Less Sociable? A Preliminary Review and Call for Research. Perspectives on Psychological Science, 13, 473-491. https://doi.org/10.1177/1745691617746509

Zhao, Y. (2014). Psychology Research of the Express Enterprise Customer Complaint Behavior. Logistics Engineering and Management, 236, 95-96. 